\title{
Les sites de hauteur des Vestins
}

Étude de l'organisation territoriale d'un peuple de l'Italie préromaine

\section{Stéphane Bourdin et Antonella Natali}

École française de Rome, Soprintendenza per i Beni Archeologici dell'Abruzzo, Université de Picardie-Jules Verne et Museo nazionale di preistoria dell'Abruzzo di Celano-Paludi (éd.)

\section{(2) OpenEdition \\ 12 Journals}

Édition électronique

URL : http://journals.openedition.org/cefr/244

DOI : $10.4000 /$ cefr.244

ISSN : 2282-5703

Éditeur

École française de Rome

Référence électronique

Stéphane Bourdin et Antonella Natali, «Les sites de hauteur des Vestins », Chronique des activités archéologiques de l'École française de Rome [En ligne], Italie centrale, mis en ligne le 19 décembre 2012, consulté le 19 avril 2019. URL : http://journals.openedition.org/cefr/244 ; DOI : 10.4000/cefr.244

Ce document a été généré automatiquement le 19 avril 2019.

(c) École française de Rome 


\title{
Les sites de hauteur des Vestins
}

\author{
Étude de l'organisation territoriale d'un peuple de l'Italie préromaine
}

\section{Stéphane Bourdin et Antonella Natali}

École française de Rome, Soprintendenza per i Beni Archeologici dell'Abruzzo, Université de Picardie-Jules Verne et Museo nazionale di preistoria dell'Abruzzo di Celano-Paludi (éd.)

1 Après quatre campagnes de terrain (2006-2009) et une campagne consacrée à l'étude du matériel déposé au laboratoire archéologique du château de Capestrano (2010), la campagne 2011, qui s'est déroulée du 11 au 30 juillet, était destinée à terminer l'inventaire et l'étude du matériel récolté lors des campagnes 2006-2007 et déposé au Museo di Preistoria dell'Abruzzo de Celano-Paludi ${ }^{1}$ et à compléter en parallèle nos observations sur le terrain.

\section{Prospections et contrôles}

2 Nous avons cette année concentré nos recherches sur le secteur d'Ocre, commune formée d'une série de frazioni, dans laquelle on voit parfois une survivance d'une organisation territoriale dispersée, de type préromain ${ }^{2}$. La colline située au NE de San Martino di Ocre, qui domine la falaise appelée Piaggia di San Panfilo et culmine à $1136 \mathrm{~m}$ d'altitude a été systématiquement inspectée. On y trouve toute une série de murs en pierres sèches, dont un enclos principal qui entoure le sommet, mais aucun matériel significatif, à l'exception de trois fragments de tuiles. Sur le versant nord, on trouve un ensemble d'enclos et de champs épierrés, désormais reconquis par la végétation. Partant de Rocca di Cambio, nous avons aussi conduit des prospections sur le rebord de plateau qui domine la colline des Coste, et porte le nom de Collalto. Là encore, le bilan est négatif, mis à part quelques tessons attestant une fréquentation pastorale. L'unique structure notable est un grand mur rectiligne, en pierres sèches à double parement, qui sépare les pâturages des communes d'Ocre et Rocca di Cambio (fig. 1). 
Fig. 1 - Les sites de hauteur des Vestins. Mur en pierres sèches marquant les confins entre les communes d'Ocre et Rocca di Cambio.

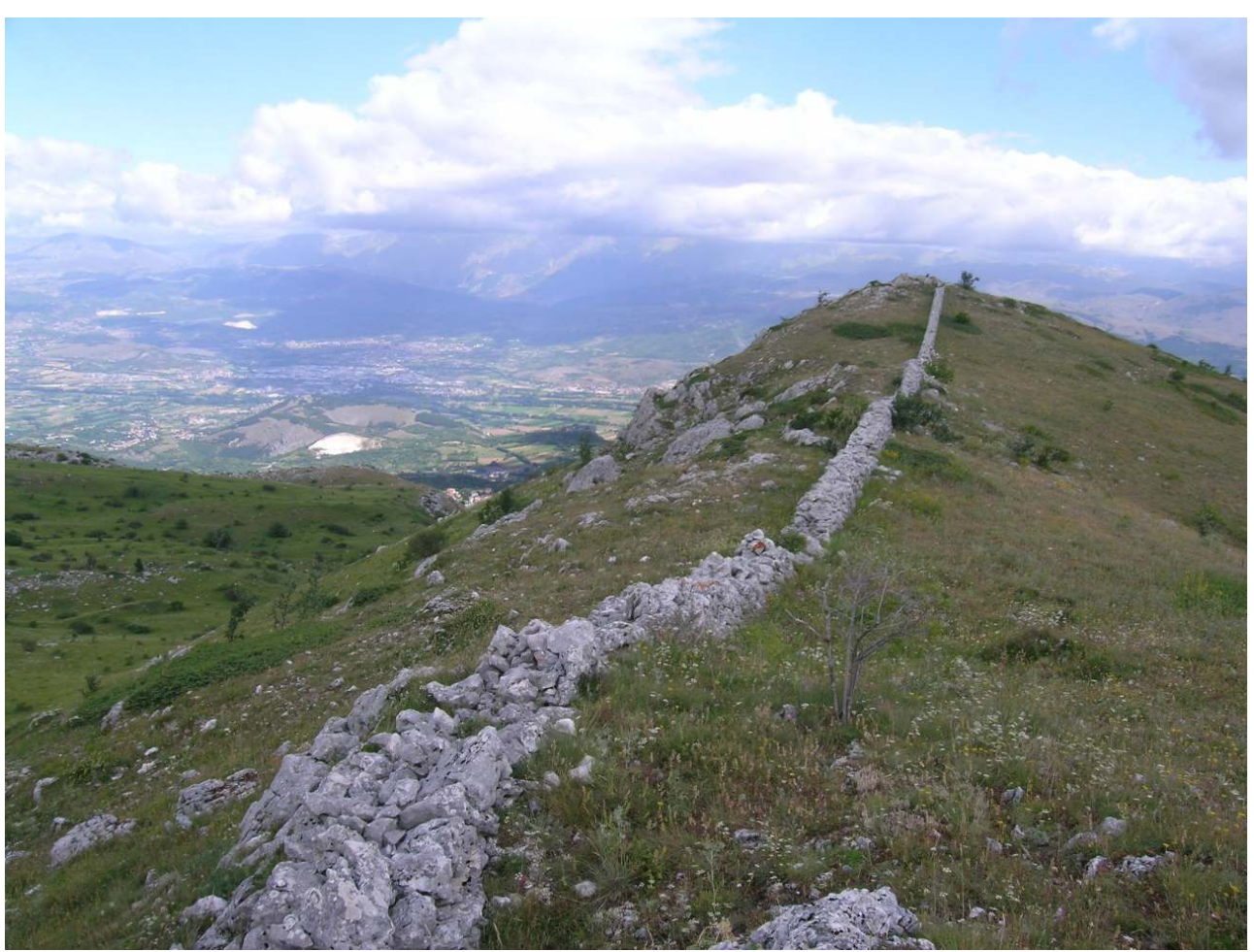

Cliché S. Bourdin.

3 Toujours sur la commune d'Ocre, mais en contrebas, nous avons inspecté la colline qui culmine à $804 \mathrm{~m}$ et domine le toponyme Santa Maria, près du bourg de Valle. Cette colline allongée possède un versant occidental en pente relativement forte et un versant oriental formé d'une falaise très raide (fig. 2 et 2 bis). 
Fig. 2 - Les sites de hauteur des Vestins. Le centre fortifié de Santa Maria (Valle, Ocre).

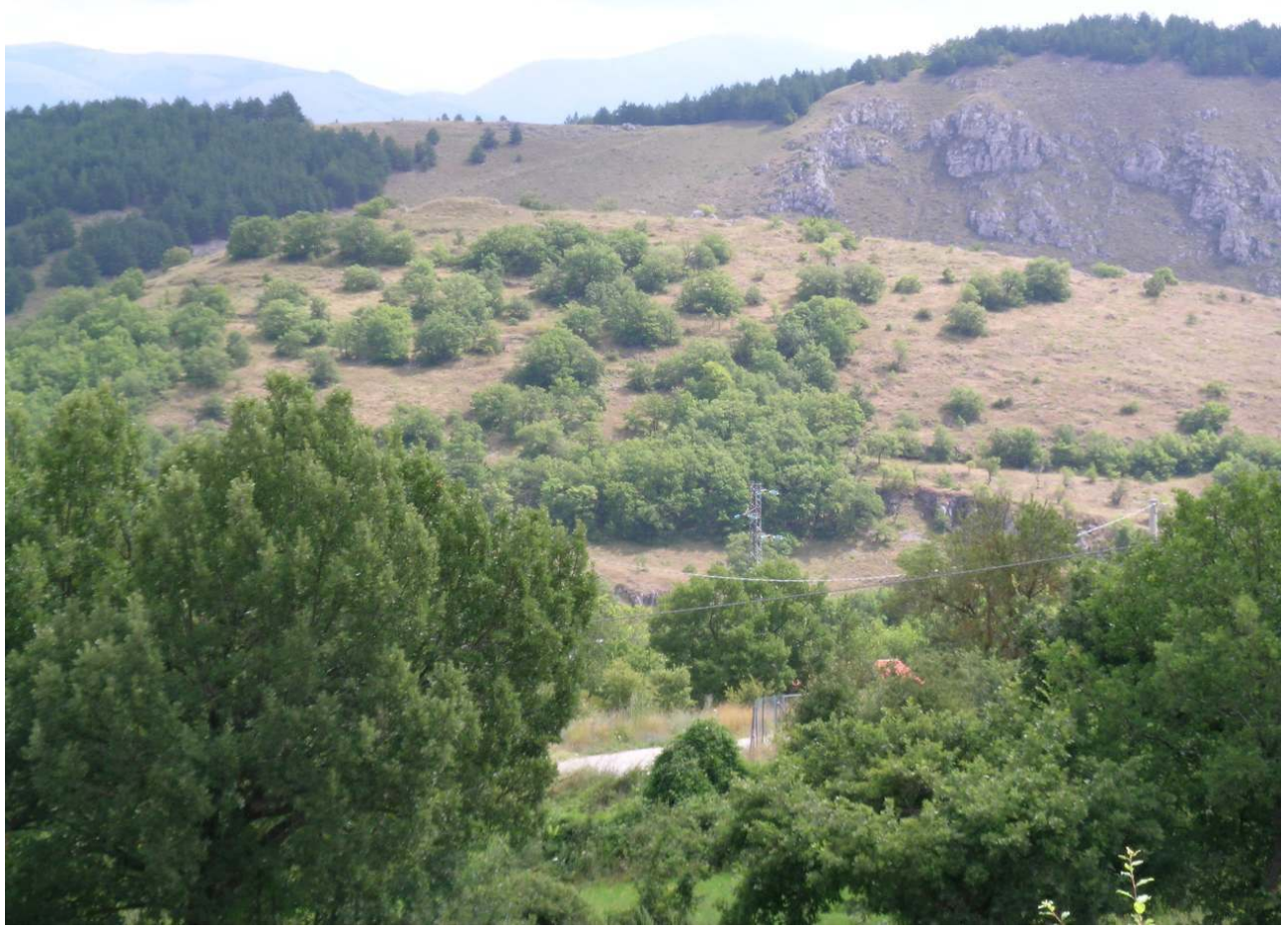

Cliché S. Bourdin.

Fig. 2 bis - Les sites de hauteur des Vestins. L'enceinte de Santa Maria (Valle, Ocre).

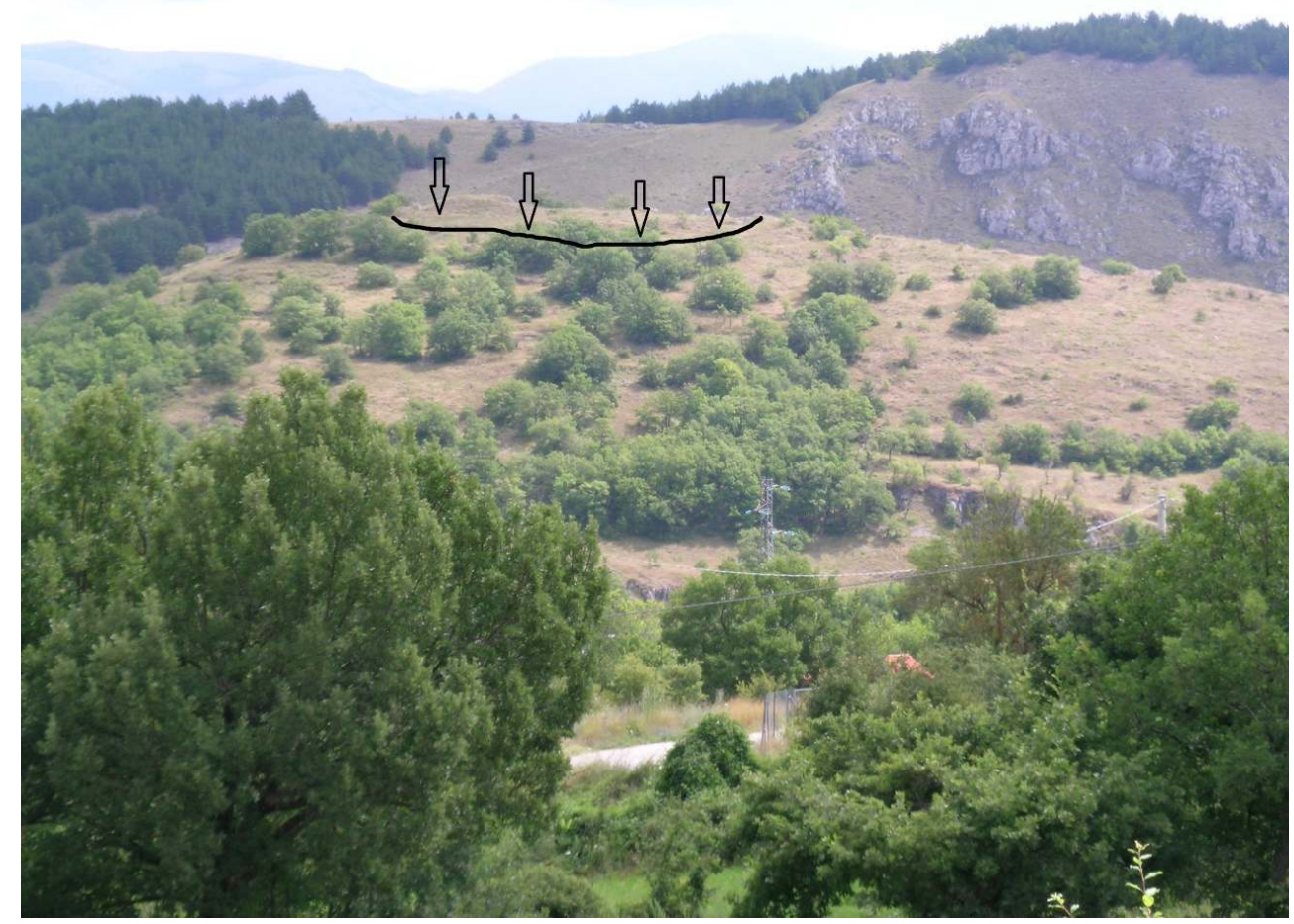

Cliché S. Bourdin. 
4 Le sommet est bordé d'une fortification, qui a été repérée, mais non décrite en détail, par A. Rapisarda, dans son récent ouvrage ${ }^{3}$. Malgré la visibilité très mauvaise, due aux herbes denses qui occupent le versant occidental, on voit clairement la présence d'un mur, avec des portions en petit appareil polygonal sur deux assises (h. max. conservée : 0,75 m), qui soutient un replat large en moyenne de 3,60 m (fig. 3).

Fig. 3 - Les sites de hauteur des Vestins. La fortification de Santa Maria.

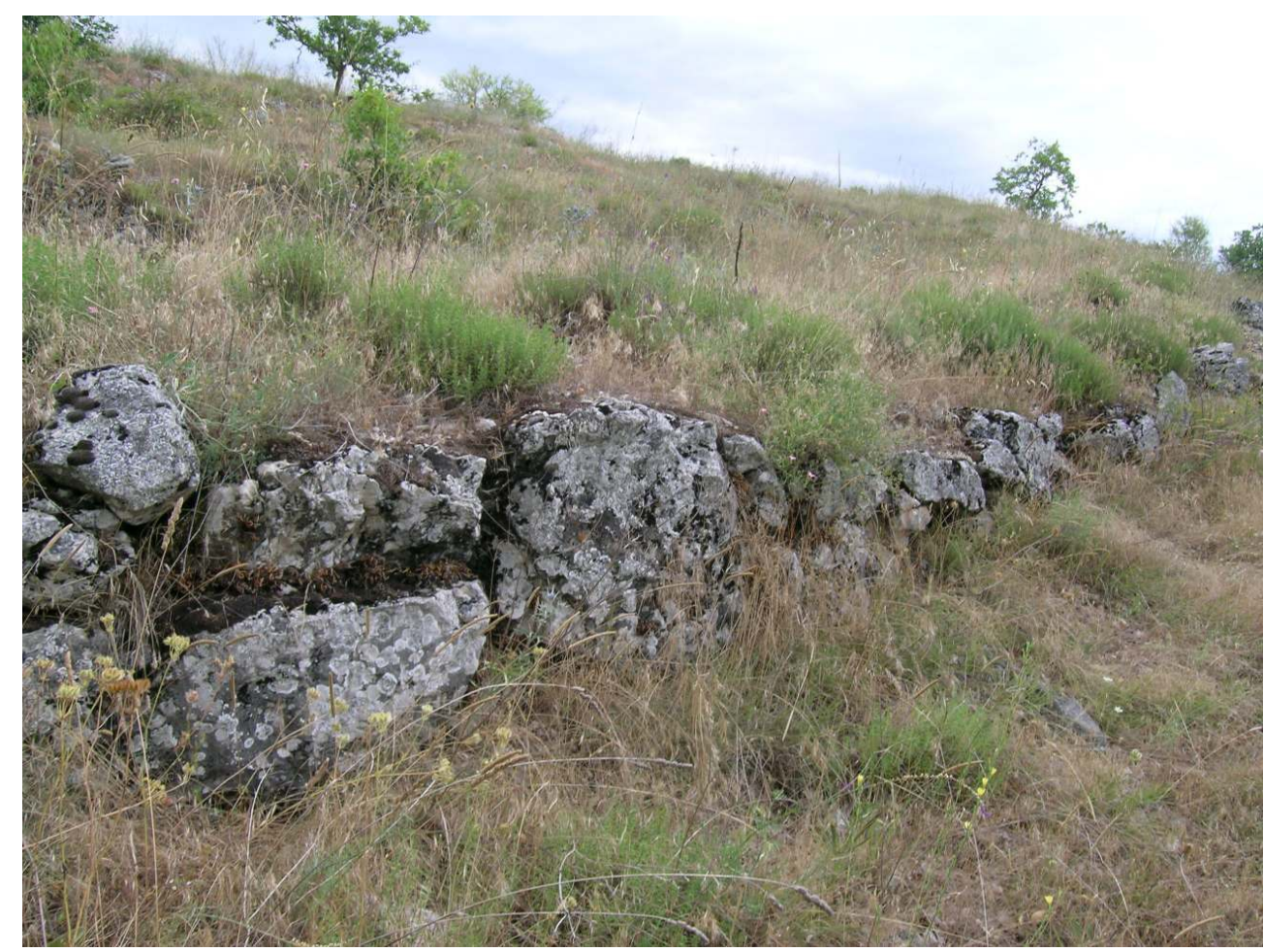

Cliché S. Bourdin.

5 Ce mur couvre tout le versant occidental, sur une longueur de $144 \mathrm{~m}$. Il s'appuie à la falaise pour fermer une surface de $3820 \mathrm{~m}^{2}$, qui a la forme d'un demi-ovale. Il s'agit donc d'un petit site, de même module que la Cote 1227 ( $0,36 \mathrm{ha})$ ou que Colle San Lorenzo $(0,32 \mathrm{ha})$, placé dans une position de contrôle d'un parcours qui reliait la plaine de L'Aquila au haut-plateau des Rocche, et surtout de toute la partie occidentale de la plaine de L'Aquila. On voit en effet depuis ce site un ensemble d'habitats antiques, comme Le Coste, le château d'Ocre, Colle San Lorenzo, Collebrincioni, Monte di Bazzano e I Colli. À l'intérieur de la surface enclose, et sur les pentes à l'extérieur, on trouve de la céramique, essentiellement des productions d'impasto du premier Âge du Fer, ce qui permet d'ajouter cette enceinte au corpus des sites fortifiés du territoire vestin.

D'autres prospections ont été conduites sur les sommets qui entourent le haut-plateau de Navelli. Partant de la Forca San Leonardo, nous avons inspecté les sommets de Monte La Serra (1223 m, commune de Carapelle Calvisio), Colle Mezzano (1166 m) et Monte Gentile (1257 m, communes de Carapelle Calvisio et San Pio delle Camere), ainsi que le vallon intermédiaire (Pompetella) sans découvrir de structure ou de matériel significatif. Le Monte Gentile, d'où l'on jouit d'un panorama impressionnant sur le haut-plateau de Navelli et sur le site de Peltuinum, tout en étant un des meilleurs points d'observation de la région, d'où l'oeil embrasse les trois secteurs principaux du territoire vestin (plaine de 
L'Aquila, haut-plateau de Navelli et vallée du Tirino), n'a donc pas livré de trace d'occupation antique. Sur la commune de San Benedetto in Perillis, nous avons inspecté le Monte Croce $(971 \mathrm{~m})$ et les environs du lac de San Benedetto. Mise à part la présence possible d'une tombe pillée près $d u$ cimetière moderne, aucune trace des murs polygonaux signalés par A. De Nino pour le toponyme «Santa Croce »" et aucun matériel n'ont pu être mis en évidence.

7 Après l'incendie de 2007, nous avions conduit de nouveau des prospections sur les centres fortifiés déjà étudiés, afin de profiter des conditions de visibilité plus grandes. Le seul centre qui restait à contrôler est le Monte Asprino $(888 \mathrm{~m})$, sur la commune de Navelli, où était signalé un replat sur le versant méridional et où nous avions récupéré du matériel, d'époque récente. Un nouvel examen a confirmé cette datation, avec la présence de céramique à surface peignée, renvoyant à l'époque médiévale. Il ne s'agit donc pas d'un centre fortifié vestin, mais d'un sommet fréquenté au Moyen Âge, en raison notamment de la très bonne visibilité sur les alentours et notamment sur le col qui consent de passer du haut-plateau de Navelli à la vallée du Tirino (Valle Iena). On peut désormais y voir de nouveau les deux cupules signalées par E. Mattiocco ${ }^{5}$ : il s'agit de petits réservoirs (diam. 31 et $18 \mathrm{~cm}$ ) creusés dans le rocher et destinés à recueillir les eaux de pluie. Le plus grand des deux possède un petit canal d'écoulement et les traces d'instrument de taille sont bien visibles en surface (fig. 4).

Fig. 4 - Les sites de hauteur des Vestins. Petits réservoirs d'eau de pluie du Monte Asprino.

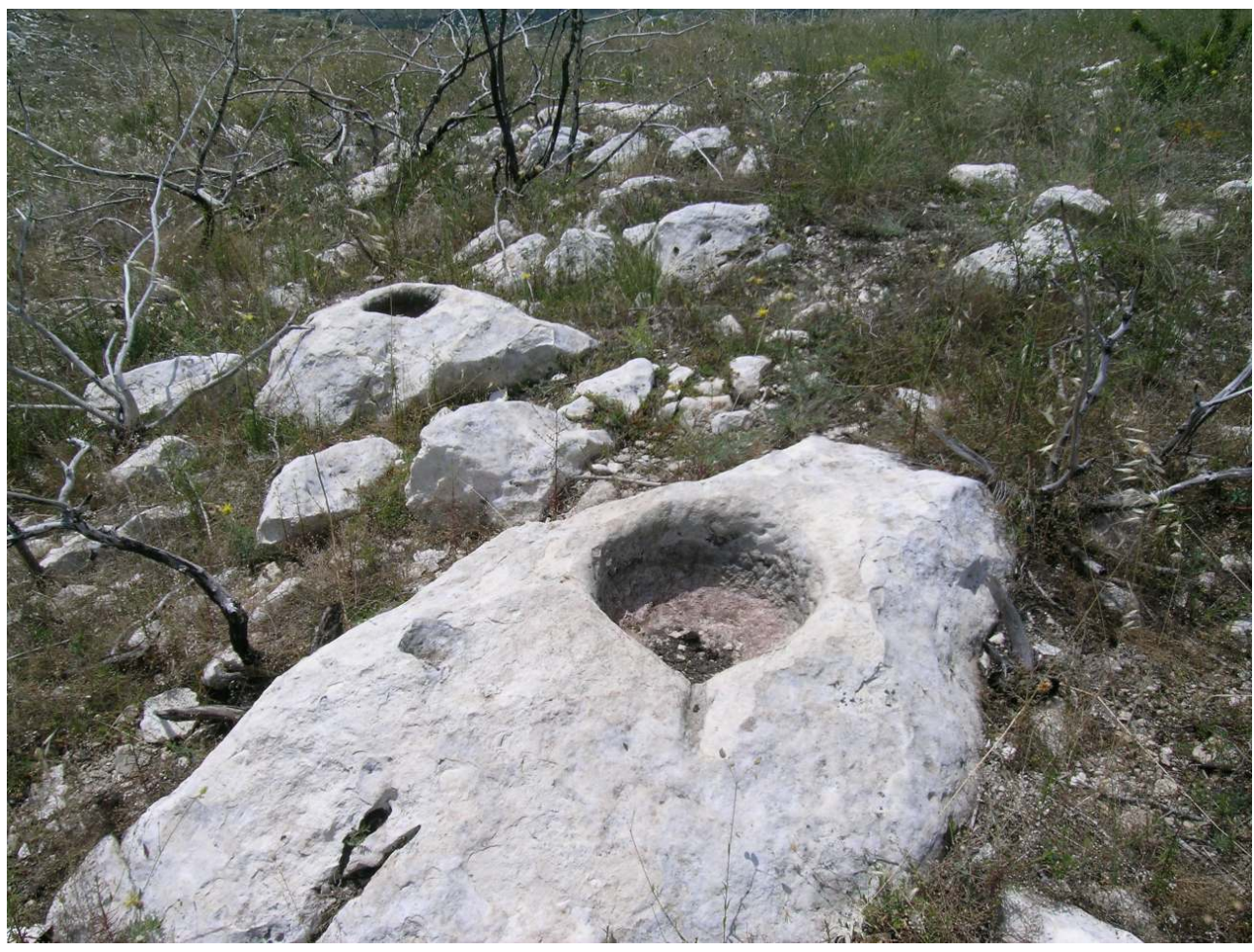

Cliché S. Bourdin.

8 Le Monte Caticchio $(635 \mathrm{~m})$ a déjà été prospecté et nous avons pu mettre en évidence la présence d'une occupation protohistorique, probablement quelques cabanes, près de son sommet, au nord. Toutefois, la partie méridionale de la colline, dite "Castellina » a depuis longtemps attiré l'attention des clandestins, mais aussi des chercheurs ${ }^{6}$. La présence 
d'une décharge perturbe la compréhension du site, mais on voit encore des portions de murs liés au mortier de chaux, des fragments de tuiles, de la céramique médiévale, mais aussi de l'impasto et en particulier un anneau de terre cuite servant probablement de lest pour un filet de pêche.

Nous avons enfin contrôlé la situation du site fortifié de Collebrincioni (1171 m, commune de L'Aquila), signalé par E. Mattiocco ${ }^{7}$. On constate la présence d'un circuit annulaire, formé d'un replat large de $6 \mathrm{~m}$ en moyenne, sans traces visibles d'un mur de soutien. Au sommet se trouvent les vestiges d'une cabane de bergers, tandis que toute la surface est parsemée de fragments de tuiles. Aucun tesson de céramique n'a en revanche pu être identifié, mais ce site est régulièrement la proie des clandestins. Il est donc difficile, en l'état actuel de nos connaissances, de préciser davantage la chronologie de l'occupation, même si le site appartient à une typologie bien connue dans la zone.

\section{L'étude du matériel ostéologique du Monte di Cerro}

10 En 2009 et 2010, plusieurs sondages ont été réalisés dans le secteur de la porte du Monte di Cerro et à l'intérieur de la surface enclose. À cette occasion, de nombreux fragments d'ossements animaux ont été récupérés. Ils proviennent, dans leur grande majorité, de niveaux de remblais, formés de terre récupérée à l'intérieur de l'habitat pour constituer un « circuit annulaire » sur lequel est implantée l'enceinte elle-même (US 1025, 1037, 1051), ou du remplissage entre les deux parements (US 1024). L'étude de ce matériel a été confiée à Alice Bourgois (doctorante à l'Université de Picardie-Jules Verne).

11 Ces sondages ont donc livré une trentaine d'ossements, dont $70 \%$ environ ont pu faire l'objet d'une identification. Il s'agit dans la très grande majorité des cas d'espèces domestiques, avec 8 restes de suidés, 6 restes de bovidés et 4 restes de caprinés. La présence d'espèces sauvages, avec en particulier un fragment d'andouiller de cerf, reste tout à fait anecdotique.

\section{Lo studio della ceramica}

12 La campagna di studio 2011 ha permesso di proseguire l'attività di classificazione del materiale ceramico intrapresa in maniera sistematica nel 2010. I frammenti analizzati sono stati rinvenuti nel corso delle campagne di ricognizione svolte nel $2006^{8}$ e nel $2007^{9}$. Lo studio è stato condotto nel mese di luglio presso i laboratori del Museo di Celano, applicando lo stesso approccio metodologico introdotto nel 2010 : la selezione per classi di materiale, il disegno dei profili dei frammenti diagnostici, la relativa quantificazione e l'elaborazione di una scheda di reperto, che accompagni la redazione del catalogo da inserire nel volume finale. Complessivamente, sono stati disegnati 193 frammenti diagnostici provenienti dalle pregresse campagne di prospezione archeologica $2006 \mathrm{e}$ 2007 e 10 frammenti relativi alla campagna 2011, per un totale di 203 disegni. Sono stati, inoltre, verificati gli inventari delle campagne 2006 e 2007 ed è stato stilato l'inventario 2011. Una preliminare revisione del materiale raccolto nel corso delle campagne $2006 \mathrm{e}$ 2007 sembra confermare e, in parte ampliare, la datazione proposta precedentemente per quel che riguarda i siti di Monte di Cerro e Colle Rischia ${ }^{10}$.

13 L'abitato fortificato di Monte di Cerro ha restituito materiali riferibili sostanzialmente alla prima età del Ferro, anche se non va esclusa una fase recenziore data la presenza di 
tegole piane con alette a profilo curvilineo ed esigui frammenti in ceramica depurata. Il sito d'altura di Colle Rischia, analogamente, conosce, oltre ad una comprovata fase ascrivibile alla prima età del Ferro, una frequentazione presumibilmente di età repubblicana-imperiale, testimoniata da frammenti di vernice nera, di sigillata italica e ceramica da fuoco ${ }^{11}$. Anche Colle Santa Rosa I si configura come centro protostorico della prima età del Ferro, data la presenza di numerosissime prese a sezione semicircolare $\mathrm{e}$ triangolare e frammenti di vasi colatoi, come nel caso di Colle Cipolla, dove compaiono olle ovoidi, prese a sezione semicircolare, scodelle, pareti con bugna e carene decorate con costolature oblique in ceramica d'impasto databili alla prima età del Ferro. Il sito d'altura denominato Quota 1052 mostra un'occupazione in età protostorica (pareti in ceramica d'impasto) ma anche in età romana, dato il ritrovamento di tegole piane e coppi frammentari, oltre ad un piede ad anello in ceramica a vernice nera, riconducibile alla fine del II-I sec. a.C.

14 A S. Pio si registra una frequentazione di età romana con elementi inequivocabilmente tardo antichi per la presenza di orli di olle in ceramica da fuoco, tipiche del periodo, una lucerna frammentaria in ceramica depurata, interpretata come imitazione locale dell'Atlante forma X e frammenti di scodelle riconducibili al tipo Hayes 61, in sigillata africana chiara D. Vanno riferiti, invece, al periodo altomedievale le pareti in ceramica da fuoco con decorazione a pettine.

Documentano una frequentazione ascrivibile ad epoca altomedievale (IX-XII sec. d.C.) i siti di Colle San Donato, Colle Sinizzo, Pizzo di Ovindoli, Le Coste, Monte Asprino, Le Cese. Il rinvenimento di fondi apodi, caratterizzati da decorazione a stuoia e pareti decorate a pettine pertinenti ad olle in ceramica da fuoco e anse a nastro, rimanda ad una sintassi decorativa ampiamente attestata in area costiera abruzzese e in maniera minoritaria nell'interno. Ceramiche affini sono testimoniate nel centro storico di Pescara, di Pianella, in quello di Colle S. Giovanni, presso Atri, e presso la cattedrale valvense di Corfinio ${ }^{12}$. Tale studio preliminare ha consentito di acquisire nuovi dati per una cronologia di quelle fasi di occupazione o, quanto meno, di frequentazione dei siti oggetto di indagine nel 2006 e 2007, confermando e ampliando, peraltro, quanto già in precedenza documentato. Tuttavia, solo in seguito ad un'analisi complessiva e dettagliata dei materiali sarà possibile tentare di definire in maniera puntuale le modalità di occupazione del territorio vestino nei vari periodi storici. [Antonella Natali]

Le matériel recueilli nous permet donc d'affirmer que la plupart des sites fortifiés signalés dans la région connaissent une occupation au premier Âge du Fer, date à laquelle sont élaborées les structures défensives, mais qu'une grande partie d'entre eux est encore occupée ou fréquentée à une époque plus récente, parfois jusqu'à l'époque romaine ou médiévale. Seules des fouilles en extension pourraient permettre toutefois de comprendre la nature de cette occupation ou de cette fréquentation. Dans les prochaines années, le travail d'étude du matériel doit être poursuivi, avec en particulier l'analyse du matériel découvert en prospection par E. Mattiocco dans les années 1980, en vue de rédiger une synthèse finale, sous la forme d'une monographie à paraître dans la Collection de l'École française de Rome. 


\section{BIBLIOGRAPHIE}

Bourdin - Natali 2008 = S. Bourdin, A. Natali, Les sites de hauteur des Vestins : étude de l'organisation territoriale d'un peuple de l'Italie préromaine, dans MEFRA, 120-1, 2008, p. 206-211.

De Nino 1982 = A. De Nino, S. Benedetto in Perillis : resti di antiche strade riconosciute nel territorio del comune ed in quello del comune prossimo di Collepietro, dans NSA, 1982, p. 484-485.

Mattiocco 1986 = E. Mattiocco, Centri fortificati vestini, Sulmone, 1986, p. 60.

Mattiocco 2006 = E. Mattiocco, Per un censimento dei centri fortificati vestini, dans E. Mattiocco (dir.), Itinera Archeologica. Contributi di archeologia abruzzese, Sulmone, 2006, p. 165-166.

Natali à paraître = A. Natali, La ceramica d'impasto dai siti d'altura vestini : campagne 2006-2009, dans S. Bourdin, V. D'Ercole (dir.), I Vestini e il loro territorio. Atti dell'Incontro di studio Nei dintorni di L'Aquila. Ricerche archeologiche nel territorio dei Vestini Cismontani prima e dopo il terremoto del 6 aprile 2009 (Rome, 12-13 febbraio 2010), à paraître.

Rapisarda 2011 = A. Rapisarda, I Vestini e il mistero del pagus di Separa, L'Aquila, 2011, p. 16-17, n $\circ 32$.

Siena - Terrigni = E. Siena, M. Terrigni, Ceramica dalla valle del Pescara. Materiali medievali dal centro storico di Pianella (Pe), dans Quaderni di Archeologia medievale, VI, 2004, p. 248-249.

Tartara $2007=$ P. Tartara, Il territorio aquilano lungo il tratturo regio. Primi dati per una carta archeologica sistematica (Area tra Bazzano e Capestrano), dans A. Clementi (dir.), I campi aperti di Peltuinum dove tramonta il sole... Saggi sulla terra di Prata d'Ansidonia dalla protostoria all'età moderna, L'Aquila, 2007, p. 469-470.

\section{NOTES}

1. Je tiens à remercier ici $\mathrm{M}^{\text {me }}$ Geltrude Di Matteo, directrice du Musée de Celano Paludi, ainsi que tout le personnel, pour l'excellent accueil que nous avons reçu et le soutien apporté à notre recherche.

2. Mattiocco 1986, p. 60.

3. Rapisarda 2011, p. 16-17, n 32. A. Rapisarda appelle ce site "San Felice d'Ocre», mais nous préférons le désigner au moyen du toponyme « Santa Maria » dont il est plus proche.

4. De Nino 1982, p. 484-485.

5. Mattiocco 1986, p. 100.

6. Tartara 2007, p. 469-470.

7. Mattiocco 2006, p. 165-166.

8. Per la campagna 2006 sono stati studiati i materiali provenienti dai siti di Monte di Cerro, Croce di Picenze, Colle del Cerchio, Colle S. Donato, Colle Separa, Monte Boria, Campo di Monte, Colle Rischia, Colle Cipolla, Le Castagne.

9. I siti di Forca Caruso, Corcumella, Leporanica, Colle Sinizzo, Collemaggiore, Colle Opi, Quota 1052, Maregelato, Castellone di Civitaretenga, i Colli, Monte di Cerro, Colle Cicogna, Le Cese, Monte Pagliaccio, Monte di Bazzano, Monte Cavalletto, Monte Callicchio, S. Pio, Le Coste, Monte 
Asprino, Pizzo di Ovindoli, Ovindoli, Serra di Navelli, Colle S. Rosa I e II sono stati oggetto d'esame per la campagna 2007.

10. Natali à paraître.

11. Bourdin - Natali 2008, p. 206-211.

12. Siena - Terrigni, p. 248-249.

INDEX

Mots-clés : préhistoire, Abruzzes, fortification, ossements, céramique

institutions École française de Rome, Soprintendenza per i Beni Archeologici dell'Abruzzo, Université de Picardie Jules Verne

\section{AUTEURS}

\section{STÉPHANE BOURDIN}

Directeur des études pour l'Antiquité, École française de Rome - dirant[at]efrome.it

\section{ANTONELLA NATALI}

Archéologue - antonellanatali78[at]gmail.com 